$8^{\text {th }}$ International conference on Research in Engineering, Science and Technology

Paris, France

November 2-4, 2018

\title{
RECUPERATION DU CATECHOL PAR DES SOLIDES DOLOMITIQUES
}

\author{
$\underline{\text { Ilhem Belarbi }}{ }^{1}$, Aouda Khalfa ${ }^{2}$, Kheira Marouf-Khelifa ${ }^{3}$, Amine Khelifa ${ }^{4}$ \\ ${ }^{1}$ Laboratoire de Structure, Elaboration et Applications des Matériaux Moléculaires (S.E.A.2M.) \\ Université de Mostaganem, Algérie.
}

\section{Résumé:}

La recherche de nouveaux matériaux, susceptibles de développer des propriétés physicochimiques intéressantes et utiles au domaine industriel, est toujours nécessaire. Parmi ces matériaux recherchés, nous retrouvons la dolomie dont l'élément essentiel est la dolomite. Il s'agit d'un minéral cristallin, formé d'un double carbonate de calcium et de magnésium, de formule $\left[\mathrm{CaMg}\left(\mathrm{CO}_{3}\right)_{2}\right]$. A l'état brut, les propriétés physicochimiques de la dolomie sont peu significatives. Néanmoins, elles peuvent être améliorées par un traitement thermique. A cet effet, ce matériau a subi une calcination à $600,800,900$ et $1000^{\circ} \mathrm{C}$, ce qui a permis d'obtenir des solides dolomitiques : D600, D800, D900, D1000.

Les différents échantillons obtenus ont servi à la récupération du catéchol. Le dosage a été effectué par spectrophotométrie UV, à la longueur d'onde caractéristique de $275 \mathrm{~nm}$. Les paramètres considérés sont le temps, le $\mathrm{pH}$ et la concentration de la solution. L'affinité d'adsorption du catéchol sur les solides dolomitiques suit la séquence suivante: D-800 > D-900 $>\mathrm{D}-1000>\mathrm{D}-600>\mathrm{D}$-brute à $25^{\circ} \mathrm{C}$. Le meilleur échantillon est le solide D-800 avec une quantité adsorbée égale à $68 \mathrm{mg} / \mathrm{g}$. Cette quantité diminue au fur et à mesure que la température augmente. Ce résultat suggère qu'il s'agit d'une physisorption.

Mots-clés: Dolomie; Traitement thermique; solides dolomitiques ; Adsorption; Catéchol.

\section{Références:}

[1] S Gunasekaran, G Anbalagan, J. Raman Spectrosc. 38 (2007) 846-852.

[2] L. Luo, L. Lou, X. Cui, B. Wu, J. Hou, B. Xun, X. Xu, Y. Chen, Journal of Hazardous Materials 185 (2011) 639-646.

[3] L. Zhou, S. Pan, X. Chen, Y. Zhao, B. Zou, M. Jin, Chemical Engineering Journal 257 (2014) 10-19.

[4] Bargar, J. R., Persson, P. \& Brown Jr, G. E. Outer-sphere adsorption of Pb(II) EDTA on goethite. Geochim. Cosmochim. Acta 63, (1999) 2957-2969.

[5] Y. Liu, M. Gao, Z. Gu, Z. Luo, Y. Ye, L. Lu, Comparison between the removal of phenol and catechol by modified montmorillonite with two novel hydroxyl-containing Gemini surfactants, Journal of Hazardous Materials 267 (2014) 71- 80. 
